\title{
QUEEN'S
UNIVERSITY
BELFAST
}

\section{Pharmacotherapeutics of capecitabine and trastuzumab in the treatment of metastatic breast cancer}

McCauley, S., Carter, G., Bennett, M., McNally, O., \& Rogers, K. (2020). Pharmacotherapeutics of capecitabine and trastuzumab in the treatment of metastatic breast cancer. British Journal of Nursing, 29(3), S4-S9. https://doi.org/10.12968/bjon.2020.29.3.S4

\section{Published in:}

British Journal of Nursing

\section{Document Version:}

Peer reviewed version

Queen's University Belfast - Research Portal:

Link to publication record in Queen's University Belfast Research Portal

\section{Publisher rights}

Copyright 2020 Mark Allen Healthcare.

This work is made available online in accordance with the publisher's policies. Please refer to any applicable terms of use of the publisher.

\section{General rights}

Copyright for the publications made accessible via the Queen's University Belfast Research Portal is retained by the author(s) and / or other copyright owners and it is a condition of accessing these publications that users recognise and abide by the legal requirements associated with these rights.

Take down policy

The Research Portal is Queen's institutional repository that provides access to Queen's research output. Every effort has been made to ensure that content in the Research Portal does not infringe any person's rights, or applicable UK laws. If you discover content in the Research Portal that you believe breaches copyright or violates any law, please contact openaccess@qub.ac.uk. 


\section{Pharmacotherapeutics of capecitabine and trastuzumab in the treatment of metastatic breast}

cancer

Sarah McCauley, Gillian Carter, Maggie Bennett, Oonagh McNally and Katherine MA Rogers Sarah McCauley, Oncology Nurse Practitioner, Ulster Hospital, Dundonald, Belfast

Gillian Carter, Lecturer in Chronic Illness, School of Nursing and Midwifery, Queen's University Belfast

Maggie Bennett, Lecturer, School of Nursing and Midwifery, Queen's University Belfast Oonagh McNally, Lecturer, School of Nursing, Ulster University, Magee Campus, Londonderry Katherine Rogers, Senior Lecturer, School of Nursing and Midwifery, Queen's University Belfast, k.rogers@qub.ac.uk

\section{Abstract}

Metastatic HER2-positive breast cancer is an incurable disease with a poor prognosis. This article presents a critical appraisal of two treatments commonly used in the treatment of metastatic HER2positive breast cancer: the oral chemotherapy drug, capecitabine, and the monoclonal antibody, trastuzumab. What follows is a critical discussion of the pharmacotherapeutics of capecitabine and trastuzumab, which considers their use both as single agents and as a combination regimen in the treatment of metastatic breast cancer. The implications of side effects of these drugs are discussed, both individually and in combination, as are the challenges these bring to the prescriber. The article evaluates the use of these agents and concludes that combination of capecitabine and trastuzumab is an attractive treatment option for patients and for the prescriber.

Key words: Breast cancer, HER2, Capecitabine, Trastuzumab, Prescribing, Systemic anti-cancer (SACT) 
The human breast develops as a result of genetic and hormonal stimulus from skin precursor cells, known as ectoderm, occurring during the fourth week of embryonic development (Sadler, 2009). Unlike most other organs, which develop to maturity at the embryonic stage, the female breast only reaches a matured functioning state in adulthood during the pregnancy-lactation cycle (Hassiotou and Geddes, 2013). It is comprised of adipose and glandular tissue supported by connective tissue called 'Cooper's ligaments' (Gefen and Dilmoney, 2007). The glandular tissue drains via the ductal system (Hassiotou and Geddes, 2013) where each duct wall consists of two layers of epithelial cells all fixed within a fibrous stroma (Visvader, 2009). The innermost layer contains cuboidal epithelial cells, some of which can transform into milksecretory cells during lactation. It is these epithelial cells where the majority of breast malignancies begin (Li et al, 2003). The exterior layer consists of contractile myoepithelial cells, which encapsulate the inner layer and have attributes of smooth muscle cells (Tezer et al, 2011).

The breast is a cutaneous secreting gland composed of skin, subcutaneous tissue, lobules, ducts and supporting tissue including fat interspaced with a complex network of lymphatics, arteries, veins, nerves and ligaments (Jesinger, 2014).

\section{Development of breast cancer}

Breast cancer begins as a local disease but it can metastasise to the lymph glands and then to distant organs, with common metastatic sites being the lungs, liver and bones (Weigelt et al, 2005). These metastatic cancer cells have features similar to the primary tumour, not the cells to where they have spread (National Cancer Institute, 2017). The protooncogene HER2 is over-expressed in $20-30 \%$ of breast cancers (Hayes and Thor, 2002), but is a poor prognostic indicator. This, in combination with negative oestrogen receptor status, has demonstrated the worst outcome in a subgroup analysis (Tsutsui et al, 2002). Once breast cancer has metastasised the disease becomes incurable and average survival ranges from 24 to 36 months (Cardoso et al, 2012). 


\section{Metastatic breast cancer treatments}

The critique here considers two treatments commonly used in metastatic HER2-positive breast cancer. Capecitabine is a cell-cycle specific, anti-metabolite chemotherapy specific to the S-phase of the cell cycle whereas trastuzumab is a targeted therapy within the monoclonal antibody classification of drugs.

\section{Capecitabine}

Capecitabine is an antineoplastic agent licensed for use as a monotherapy for patients with metastatic breast cancer in whom treatment with an anthracycline and taxane chemotherapy regime has failed (Joint Formulary Committee, 2020). Capecitabine is a fluoropyrimidine carbamate, a prodrug of the antimetabolite 5-fluorouracil (5-FU) (Lam et al, 2016) and affects protein synthesis and RNA processing (Law et al, 2015). Capecitabine is transformed to 5-FU initially in the liver with the final transformation occurring by the enzymes cytidine deaminase and thymidine phosphorylase (Law et al, 2015), found in tumour tissues and to a lesser extent normal tissues (Accord-UK Ltd, 2017). It interferes with the synthesis of DNA by obstructing the action of the enzyme thymidylate synthetase by acting as a false pyrimidine base (Law et al, 2015). Research demonstrates that the metabolism of 5-FU in the anabolic pathway prevents the methylation reaction of deoxyuridylic acid to thymidylic acid, therefore impeding DNA synthesis (Accord-UK Ltd, 2017). It is proposed that 5-FU may act by generating a thymidine deficiency that incites unstable development and apoptosis of the cancer cell (Accord-UK Ltd, 2017).

Capecitabine is administered orally, and is rapidly and expansively absorbed (Accord-UK Ltd, 2017), before being converted to the metabolites 5'-deoxy-5-flurocytidine (5'-DFCR) and 5'-deoxy5-fluororidine (5'-DFUR). Effective absorption of capecitabine can be negatively impacted if administered with food (Singh and Malhotra, 2004), specifically, the rate of absorption is decreased, however, this results only in a slight effect on the area under the curve (AUC) of 5'-DFUR and of the resultant metabolite 5-FU (Accord-UK Ltd, 2017). Consequently, it is recommended that 
capecitabine should be taken 30 minutes after meals (Roche Products Ltd 2019a).

In vitro studies demonstrated that capecitabine is metabolised to $5^{\prime}$-DFCR by hepatic carboxylesterase, before being transformed to $5^{\prime}$-DFUR by cytidine deaminase, principally located in the liver and tumour cells (Accord-UK Ltd, 2017). Thymidine phosphorylase then creates further catalytic activation of 5'-DFUR intracellularly (Schellens, 2007). The biological transformation of capecitabine to 5-FU results in greater concentrations of it within tumour tissues. It

is then broken down by dihydropyrimidine dehydrogenase (DPD) to dihydro-5-fluorouracil, which is less toxic; a deficiency in DPD may lead to increased capecitabine toxicity (Accord-UK Ltd, 2017). Currently, in the UK, most NHS patients are not routinely tested for DPD deficiency before starting treatment, mainly because the effectiveness of such tests is variable. Capecitabine is predominantly eliminated as metabolites via the urine, with a half-life of less than 1 hour (Schellens, 2007). Capecitabine has been examined as both a monotherapy and in combination with other chemotherapies in the palliation of metastatic breast cancer. Results from phase 2 randomised controlled trials indicated that when capecitabine is used as a monotherapy a response is seen in approximately $20 \%$ of patients, these responses were related to symptom management and prolonged survival (Summerhayes, 2002). In particular, Blum et al's (1999) phase 2 clinical trial of 163 patients demonstrated the therapeutic ability of capecitabine in metastatic breast cancer. Participants in this study had been previously treated with several chemotherapy regimens. The results demonstrated a median survival of 12.8 months, corroborated by other research (Cervantes et al, 2000; Watanabe et al, 2001).

Capecitabine has known dose-limiting toxicities, including palmar-plantar erythrodysesthesia (handfoot syndrome), diarrhoea, abdominal pain, nausea and stomatitis (Accord-UK Ltd, 2017) and neutropenia (Roche Pharmaceuticals 2019a; Joint Formulary Committee, 2020). Cardiotoxicity has been associated with fluoropyrimidine therapy (Shah et al, 2012) although the mechanism 
for this is poorly understood (Polk et al, 2013), therefore capecitabine needs to be used with caution in patients who have a history of cardiovascular disease (Joint Formulary Committee, 2020). Caution is also advised when treating patients with renal impairment. Analysis of safety data presented an increased frequency of grade three and four adverse reactions related to treatment, in comparison with patients who had normal renal function (Accord-UK Ltd, 2017).

Furthermore, careful monitoring of patients with minor hepatic impairment is recommended, with avoidance of capecitabine therapy in cases of severe hepatic impairment (Joint Formulary Committee, 2020). Finally, capecitabine is contraindicated in patients with DPD deficiency (Joint Formulary Committee, 2020) triggered by mutations in the DPYD gene (Kuilenburg et al, 2016). Such patients have the greatest risk of fatal toxicity or life-threatening toxicity and must not be treated with capecitabine (Accord-UK Ltd, 2017).

The most common side-effect associated with capecitabine is hand-foot syndrome (Michaud and Jones, 2002). Erythema develops on the soles of the feet and palms of the hands with patients experiencing pain, tingling and numbness. If capecitabine is continued despite these side-effects, patients may develop severe blistering and exfoliation of both the palms and soles, with a significant negative impact on their activities of daily living (Michaud and Jones, 2002). Studies have demonstrated that the development of hand-foot syndrome may in fact be a clinical indicator of improved survival for patients (Hofheinz et al, 2012; Twelves et al, 2012). Hofheinz et al (2012) highlighted that patients who develop hand-foot syndrome also have a higher propensity to develop diarrhoea, stomatitis/mucositis and fatigue, however, they did not have more adverse haematological events than patients who did not develop hand-foot syndrome. Hofheinz et al (2012) speculated that mucosal tissue is more susceptible to elevated plasma concentrations of capecitabine and its metabolites than the haematopoietic system. Trials have failed to identify supportive or prophylactic treatments that can be effective in preventing or alleviating hand-foot syndrome (Kang et al, 2010;Wolf et al, 2010). Current recommendations are to interrupt treatment 
until symptoms improve then restart with a dose reduction (Martin et al, 2015; Roche Pharmaceuticals, 2019a; Joint Formulary Committee, 2020).

Diarrhoea is known to affect a significant proportion (50\%) of patients taking capecitabine (AccordUK Ltd, 2017). Dranitsaris et al (2015) reported that although patients taking capecitabine had fewer side-effects in comparison with other cytotoxic agents, the most prominent side-effects (diarrhoea and hand-foot syndrome), led to a greater proportion of patients requiring discontinuation of treatment in comparison with other treatments. Study results imply that although capecitabine is generally well tolerated, if side-effects do occur they can be difficult to manage therefore necessitating discontinuation of treatment (Dranitsaris et al, 2015).

Summerhayes (2002) noted that few studies had evaluated the interaction between capecitabine and non-oncology drugs, but highlighted that Maalox, an antacid containing magnesium hydroxides and aluminium, can significantly affect the absorption of capecitabine. Tyler (2011) made the same point regarding Maalox and also noted that the effects of warfarin and phenytoin can be elevated when co-administered with capecitabine. Leucovorin can increase the concentration of 5fluorouracil therefore risking toxicity (Tyler, 2011). The British National Formulary (BNF) (Joint Formulary Committee, 2020) cautions that allopurinol should not be used alongside capecitabine as it interferes with the effectiveness of capecitabine. Capecitabine should be avoided in pregnancy as it is teratogenic, and breast feeding should be discontinued (Joint Formulary Committee, 2020). Discontinuation of capecitabine is usually due to disease progression rather than because of sideeffects (Rajora et al, 2001). Where any interaction is suspected, the current BNF should be consulted for up-to-date prescribing information.

\section{Trastuzumab}

Trastuzumab is a humanized monoclonal antibody that targets the HER2 receptor protein (Michaud and Jones, 2002). The HER2 gene is an important predictor of both time to relapse and overall 
survival in breast cancer patients (Slamon et al, 1987). Artac et al (2014) highlighted that overexpression of HER2 is associated with poor outcomes. Trastuzumab has been shown to inhibit tumour growth both when used alone and in conjunction with some chemotherapies (Michaud and Jones, 2002). Treatment with trastuzumab has been demonstrated to be effective and well tolerated (Boekhout et al, 2011); in a phase 3 trial in which it was used in combination with chemotherapy, overall response rate, survival and time to disease progression significantly improved when compared with chemotherapy alone (Brufsky, 2010). Furthermore, trastuzumab is indicated for the treatment of adult patients with metastatic breast cancer that expresses HER2 (Roche Products Ltd, 2019b). Patients with HER2-positive metastatic tumours may benefit from treatment with trastuzumab as a single agent, or in combination with chemotherapy (Jelovac and Emens 2013). HER2 testing is mandatory before treatment with trastuzumab can be initiated (Roche Products Ltd, 2019b). Originally trastuzumab was given as an intravenous infusion, however, a subcutaneous version was later developed. The HannaH trial (Jackisch et al, 2016) was a phase 3, randomised, open-label, multicentre study that evaluated the efficacy and safety of subcutaneous trastuzumab compared with that of intravenous trastuzumab. Comparable efficacy was demonstrated between intravenous administration and subcutaneous administration (Roche, 2012; Jackisch et al, 2016). In the metastatic setting patients being treated with trastuzumab should remain on treatment until progression of the disease (Roche Products Ltd, 2019b).

Trastuzumab (in its subcutaneous form) includes recombinant human hyaluronidase (rHuPH20), an enzyme used to increase its dispersion and absorption (Roche Products Ltd, 2019b). Trastuzumab is a recombinant humanised IgG1 (immunoglobulin G1) monoclonal antibody that binds selectively with sub-domain IV, which is a juxtamembrane area of HER2's extracellular domain (Roche Products Ltd, 2019b). Trastuzumab's mechanism of action is poorly understood (Michaud and Jones, 2002), however, it is believed that trastuzumab works by suppressing the HER2-receptor protein therefore hindering the downstream signal from this receptor (Sliwkowski et al, 1999). The immune system 
can locate and kill tumour cells and as such trastuzumab enhances this pathway. It has been shown in both animal and in vitro studies to impede the propagation of cancer cells that overexpress HER2, and has also been demonstrated as a powerful mediator of antibody-dependant cell-mediated toxicity, a mechanism of cell-mediated immune defence (Iannello and Ahmad, 2005; Roche Products Ltd, 2019b).

Monoclonal antibodies are administered parenterally due to limited gastrointestinal stability, large molecule size and poor lipophilicity (Wang et al, 2016). Absorption following subcutaneous administration is enabled by the lymphatic system with bioavailability ranging from $50 \%$ to $80 \%$ (Lobo et al, 2004). Absorption rate is slow when administered subcutaneously, taking a number of days to reach peak serum levels (Wang et al, 2016). The distribution of monoclonal antibodies is complex, however, convection is believed to be the primary mechanism for the transfer of antibodies to the vasculature from the interstitial spaces, with other factors such as binding affinity, antibody kinetics and receptor expression levels directly affecting antibody distribution throughout the body (Lobo et al, 2004). Monoclonal antibodies are not excreted via the renal or hepatic systems, instead they undergo proteolytic catabolism, being broken down into amino acids and peptide fragments, which can be used for protein synthesis or as energy supply (Wang et al, 2016). Elimination of trastuzumab in humans is complex but is facilitated by epithelial cells, with trastuzumab binding to HER2 and being metabolised intracellularly resulting in nonlinear elimination, demonstrating a half-life of approximately 28 days (Boekhout et al, 2011). The main side-effects of trastuzumab are cardiac dysfunction and administration-related reactions (Michaud and Jones, 2002; Roche Products Ltd, 2019b). Cardiotoxicity was, according to Michaud and Jones (2002), first reported when anthracycline chemotherapy was given in conjunction with trastuzumab. Although congestive heart failure had been noted with anthracycline monotherapy, in cases where trastuzumab was part of the drug regimen, cardiac events were seen much earlier in the course of treatment and in a higher proportion. Roche Products Ltd (2019b) reports that cardiac 
events have presented in patients receiving only trastuzumab, or trastuzumab in combination with a taxane chemotherapy, especially following treatment with anthracyclines. Therefore, all patients eligible for trastuzumab must have baseline cardiac assessments performed every 3 months during treatment and every 6 months following withdrawal of trastuzumab until 24 months after the final treatment (Roche Products Ltd, 2019b; Joint Formulary Committee, 2020). In patients receiving trastuzumab whose left ventricular ejection fraction percentage drops either 10 points from baseline or below $50 \%$, treatment should be deferred and a repeat echocardiogram performed within 3 weeks (Roche Products Ltd, 2019b). According to Ewer et al (1999) and Roche Products Ltd (2019b) cardiotoxicity with trastuzumab is unique, if it is detected early and treated promptly, patients can resume treatment with trastuzumab. Of note, trastuzumab should not be given concomitantly with anthracycline chemotherapy in the metastatic setting because of the excessive rates of cardiotoxicity (Montemurro, 2014; Roche Products Ltd, 2019b; Joint Formulary Committee, 2020). Subcutaneous administration-related reactions have been reported with trastuzumab (Roche Products Ltd, 2019b). Serious administration-related reactions such as respiratory distress, hypotension and tachycardia were not reported in clinical trials with subcutaneous administration, however, clinicians should be cautious as they are reported with the intravenous version (Richards et al, 2011; Roche Products Ltd, 2019b). Consequently, the summary of product characteristics for Herceptin (Roche Products Ltd, 2019b) recommends caution when using the subcutaneous version of trastuzumab as serious pulmonary events have been described with the drug's intravenous version.

Animal studies using Cynomolgus monkeys identified no reduction in fertility or harm to the foetus, however, the transfer of trastuzumab across the placenta was noted in early and late gestation, and although animal studies cannot always predict the response in humans, trastuzumab should be avoided in pregnancy unless the potential benefit for the mother outweighs the potential risk to the foetus (Roche Products Ltd, 2019b). Women who become pregnant while taking trastuzumab should be counselled regarding potential harm to the foetus. In the post-marketing phase of trastuzumab 
there were reports of renal growth and function impairment associated with a deficiency in amniotic fluid, and some related fatal pulmonary hypoplasia in the foetuses of women who were pregnant and receiving trastuzumab (Roche Products Ltd, 2019b). It is unknown whether trastuzumab is transferred in human breast milk, therefore breast feeding is contraindicated during treatment and for 7 months after the last dose (Roche Products Ltd, 2019b; Joint Formulary Committee, 2020).

\section{Combination of trastuzumab and capecitabine}

Schaller et al (2007) reported that the combination of trastuzumab and capecitabine was extremely active in patients with metastatic HER2-overexpressing breast cancer who have been treated with taxanes and anthracyclines. Ishida et al (2009) examined the activity of capecitabine and trastuzumab in patients with metastatic HER2-positive breast cancer and who were resistant to both anthracycline and taxane chemotherapies. Participants received six cycles of treatment and if toxicity developed with capecitabine, treatment was delayed and a dose reduction initiated, however, dose modification of trastuzumab was prohibited (Ishida et al, 2009). Antiemetics and antidiarrheal drugs could be administered as necessary, but trastuzumab was discontinued if cardiotoxicity developed (Ishida et al, 2009). The study found that median overall survival was 22.3 months when capecitabine and trastuzumab were combined, compared with 12.8 months where capecitabine was used alone. This suggests that the combination of capecitabine and trastuzumab can increase overall survival for patients. Ishida et al (2009) concurred with Schaller et al (2007) that the combination of capecitabine and trastuzumab is well-tolerated with hand-foot syndrome, pain and gastrointestinal symptoms being the most common adverse events, but these appear to have been well controlled with adjuncts to treatment. Ishida et al did not report any treatment-related deaths. 


\section{Conclusion}

Capecitabine and trastuzumab have different modes of action but are used for the same disease process. Trials demonstrated the efficacy of both these treatments in the palliation of metastatic breast cancer when used as monotherapy (Blum et al, 1999; Cervantes et al, 2000; Watanabe et al, 2001; Boekhout et al, 2011). Ishida et al's study (2009) demonstrated the benefit to overall survival when these drugs are administered concurrently. Considering the palliative nature of metastatic breast cancer, the benefit to overall survival when using both capecitabine and trastuzumab cannot be understated. Trials have demonstrated that although neither of these drugs will cure metastatic breast cancer they are effective in slowing the disease process and alleviating symptoms while being generally well tolerated. Both are easily administered, reducing patients' time in hospital when compared to the intravenous alternatives. The combination of capecitabine and trastuzumab is certainly an attractive treatment option both for patients and the prescriber.

\section{References}

Accord-UK Ltd. Capecitabine Actavis 500mg film-coated tablets. Summary of product characteristics. 2017. https://www.medicines.org.uk/emc/ medicine/28428 (accessed 22 January 2020)

Artac M, Koral L, Toy H, Guler T, Boruban MC, Altundag K. Complete response and long-term remission to anti-HER2 combined therapy in a patient with breast cancer presented with bone marrow metastases. J Oncol Pharm Pract. 2014;20(2):141-145.

https://doi.org/10.1177/1078155213480201

Blum JL, Jones SE, Buzdar AU. Multicenter phase II study of capecitabine in paclitaxel-refractory metastatic breast cancer. J Clin Oncol. 1999;17(2):485-493.

https://doi.org/10.1200/JCO.1999.17.2.485 
Boekhout AH, Beijnen JH, Schellens JHM. Trastuzumab. Oncologist. 2011;16(6):800-810.

https://doi.org/10.1634/theoncologist.2010-0035

Brufsky A. Trastuzumab-based therapy for patients with HER2-positive breast cancer: from early scientific development to foundation of care. Am J Clin Oncol. 2010;33(2):186-195

Cardoso F, Costa A, Norton L et al. 1st International consensus guidelines for advanced breast cancer (ABC 1). Breast. 2012;21(3):242-252. https://doi.org/10.1016/i.breast.2012.03.003

Cervantes G, Torrecillas L, Erazo AA, et al. Capecitabine (Xeloda) as treatment after failure to taxanes for metastatic breast cancer. Proceedings of the American Society of Clinical Oncology. 2000; 19:121a. Abstract 469

Dranitsaris G, Beegle N, Kalberer T, Blau S, Cox D, Faria C. A comparison of toxicity and health care resource use between eribulin, capecitabine, gemcitabine, and vinorelbine in patients with metastatic breast cancer treated in a community oncology setting. J Oncol Pharm Pract.

2015;21(3):170-177. https://doi.org/10.1177/1078155214525369

Ewer MS, Gibbs HR, Swafford J, Benjamin RS. Cardiotoxicity in patients receiving trastuzumab (Herceptin): primary toxicity, synergistic or sequential stress, or surveillance artifact? Semin Oncol. 1999;26(4 Suppl 12):96-101

Gefen A, Dilmoney B. Mechanics of the normal woman's breast. Technol Health Care. 2007;15(4):259-271. https://doi.org/10.3233/THC-2007-15404

Hassiotou F, Geddes D. Anatomy of the human mammary gland: current status of knowledge. Clin Anat. 2013;26(1):29-48. https://doi.org/10.1002/ca.22165

Hayes DF, Thor AD. c-erbB-2 in breast cancer: development of a clinically useful marker. Semin Oncol. 2002;29(3):231-245. https://doi.org/10.1053/sonc.2002.32899 
Hofheinz R-D, Heinemann V, von Weikersthal LF et al. Capecitabineassociated hand-foot-skin reaction is an independent clinical predictor of improved survival in patients with colorectal cancer. Br J Cancer. 2012;107(10):1678-1683. https://doi.org/10.1038/bjc.2012.434

lannello A, Ahmad A. Role of antibody-dependent cell-mediated cytotoxicity in the efficacy of therapeutic anti-cancer monoclonal antibodies. Cancer Metastasis Rev. 2005;24(4):487-499. https://doi.org/10.1007/s10555-005-6192-2

Ishida T, Kiba T, Takeda M et al. Phase II study of capecitabine and trastuzumab combination chemotherapy in patients with HER2 overexpressing metastatic breast cancers resistant to both anthracyclines and taxanes. Cancer Chemother Pharmacol. 2009;64(2):361-369.

https://doi.org/10.1007/s00280-008-0882-8

Jackisch C, Hegg R, Stroyakovskiy D et al. HannaH phase III randomised study: association of total pathological complete response with event-free survival in HER2-positive early breast cancer treated with neoadjuvant- adjuvant trastuzumab after 2 years of treatment-free follow-up. Eur J Cancer. 2016;62:62-75. https://doi.org/10.1016/i.ejca.2016.03.087

Jelovac D, Emens LA. HER2-directed therapy for metastatic breast cancer. Oncology (Williston Park). 2013;27(3):166-175. https://tinyurl.com/qqqo84s

Jesinger RA. Breast anatomy for the interventionalist. Tech Vasc Interv Radiol. 2014;17(1):3-9. https://doi.org/10.1053/i.tvir.2013.12.002

Joint Formulary Committee. British National Formulary online. 2020. https://bnf.nice.org.uk (accessed 27 January 2020)

Kang YK, Lee SS, Yoon DH et al. Pyridoxine is not effective to prevent hand-foot syndrome associated with capecitabine therapy: results of a randomized, double-blind, placebo-controlled study. J Clin Oncol. 2010;28(24):3824-3829. https://doi.org/10.1200/JCO.2010.29.1807 
Kuilenburg ABP, Meijer J, Tanck MWT et al. Phenotypic and clinical implications of variants in the dihydropyrimidine dehydrogenase gene. Biochimica et Biophysica Acta (BBA) - Molecular Basis of Disease. 2016;1862(4):754-762. https://doi.org/10.1016/j.bbadis.2016.01.009

Lam SW, Guchelaar HJ, Boven E. The role of pharmacogenetics in capecitabine efficacy and toxicity. Cancer Treat Rev. 2016;50:9-22. https://doi.org/10.1016/i.ctrv.2016.08.001

Law A, Dyson S, Anthony D. An exploratory study to identify risk factors for the development of capecitabine-induced Palmar Plantar Erythrodysesthesia (PPE). J Adv Nurs. 2015;71(8):1825-1832. https://doi.org/10.1111/jan.12639

$\mathrm{Li} \mathrm{Cl}$, Moe RE, Daling JR. Risk of mortality by histologic type of breast cancer among women aged 50 to 79 years. Arch Intern Med. 2003;163(18):2149-2153.

https://doi.org/10.1001/archinte.163.18.2149

Lobo ED, Hansen RJ, Balthasar JP. Antibody pharmacokinetics and pharmacodynamics. J Pharm Sci. 2004;93(11):2645-2668. https://doi.org/10.1002/ips.20178

Martín M, Martínez N, Ramos M et al. Standard versus continuous administration of capecitabine in metastatic breast cancer (GEICAM/2009-05): a randomized, noninferiority phase II trial with a pharmacogenetic analysis. Oncologist. 2015;20(2):111-112.

https://doi.org/10.1634/theoncologist.2014-0379

Michaud LB, Jones KL. Recent advances in the systemic management of metastatic breast cancer. Journal of Pharmacy Practice. 2002;15(1):52-61. https://doi.org/10.1106/WFQY-VTFL-WLMC-TPE0 Montemurro F. Should trastuzumab be administered concomitantly with anthracycline in women with early, HER2-positive breast cancer? Transl Cancer Res. 2014;3(6):541-546.

National Cancer Institute. Metastatic cancer. 2017. http://www.cancer.gov/types/metastatic-cancer (accessed 22 January 2020) 
Polk A, Vaage-Nilsen M, Vistisen K, Nielsen DL. Cardiotoxicity in cancer patients treated with 5fluorouracil or capecitabine: A systematic review of incidence, manifestations and predisposing factors. Cancer Treat Rev. 2013;39(8):974-984. https://doi.org/10.1016/i.ctrv.2013.03.005

Rajora A, Chow KS, Vu TT, Chambers CR. Documentation of capecitabine usage as a third-line chemotherapy option for metastatic breast cancer patients. J Oncol Pharm Pract. 2001;6(4):139145. https://doi.org/10.1177/107815520100600402

Richards D, Coleman J, Reynolds J, Aronson J. Oxford handbook of practical drug therapy. 2nd edn. Oxford: Oxford University Press; 2011

Roche. Roche's Herceptin given by subcutaneous injection offers greater convenience to patients and reduces overall healthcare costs compared to standard IV infusion Investor update. 3 March 2012. https://tinyurl.com/w9tz2xk (accessed 22 January 2020)

Roche Products Ltd. Xeloda (capecitabine) 150mg film-coated tablets/500mg film-coated tablets. Patient information leaflet. 2019a. https://www.medicines.org.uk/emc/product/1319/pil (accessed 22 January 2020)

Roche Products Ltd. Herceptin 600mg solution for injection in vial. Summary of product characteristics. 2019b. https://www.medicines.org.uk/emc/medicine/28179 (accessed 22 January 2020)

Sadler TW. Langman's medical embryology. 11th edn. Baltimore: Lippincott Williams and Wilkin; 2009

Schaller G, Fuchs I, Gonsch T et al. Phase II study of capecitabine plus trastuzumab in human epidermal growth factor receptor 2 overexpressing metastatic breast cancer pretreated with anthracyclines or taxanes. J Clin Oncol. 2007;25(22):3246-3250.

https://doi.org/10.1200/JCO.2006.09.6826 
Schellens JHM. Capecitabine. Oncologist. 2007;12(2):152-155.

https://doi.org/10.1634/theoncologist.12-2-152

Shah NR, Shah A, Rather A. Ventricular fibrillation as a likely consequence of capecitabine-induced coronary vasospasm. J Oncol Pharm Pract. 2012;18(1):132-135.

https://doi.org/10.1177/1078155211399164

Tsutsui S, Ohno S, Murakami S, Hachitanda Y, Oda S. Prognostic value of c-erbB2 expression in breast cancer. J Surg Oncol. 2002;79(4):216-223. https://doi.org/10.1002/jso.10079

Singh BN, Malhotra BK. Effects of food on the clinical pharmacokinetics of anticancer agents: underlying mechanisms and implications for oral chemotherapy. Clin Pharmacokinet. 2004;43(15):1127-1156. https://doi.org/10.2165/00003088-200443150-00005 Slamon D, Clark G, Wong S, Levin W, Ullrich A, McGuire W. Human breast cancer: correlation of relapse and survival with amplification of the HER-2/neu oncogene. Science. 1987;235(4785):177182. https://doi.org/10.1126/science. 3798106

Sliwkowski MX, Lofgren JA, Lewis GD, Hotaling TE, Fendly BM, Fox JA. Nonclinical studies addressing the mechanism of action of trastuzumab (Herceptin). Semin Oncol. 1999;26(4 Suppl 12):60-70

Summerhayes M. Capecitabine: a novel, orally administered, tumour-activated treatment for breast cancer. J Oncol Pharm Pract. 2002;8(1):1-17. https://doi.org/10.1191/1078155202jp086oa

Tezer M, Bakkalog`lu H, Ergüven M, Bilir A, Kadiog`lu A. Smooth muscle morphology in the nippleareola complex. J Morphol Sci. 2011;28(3):171-175

Twelves C, Scheithauer W, McKendrick J. Capecitabine versus 5-fluorouracil/folinic acid as adjuvant therapy for stage III colon cancer: final results from the X-ACT trial with analysis by age and preliminary evidence of a pharmacodynamic marker of efficacy. Ann Oncol. 2012;23(5):1190-1197. https://doi.org/10.1093/annonc/mdr366 
Tyler T. Drug interactions in metastatic breast cancer. J Oncol Pharm Pract. 2011;17(3):236-245. https://doi.org/10.1177/1078155210379167

Visvader JE. Keeping abreast of the mammary epithelial hierarchy and breast tumorigenesis. Genes Dev. 2009;23(22):2563-2577. https://doi.org/10.1101/gad.1849509

Wang J, Iyer S, Fielder PJ, Davis JD, Deng R. Projecting human pharmacokinetics of monoclonal antibodies from nonclinical data: comparative evaluation of prediction approaches in early drug development. Biopharm Drug Dispos. 2016;37(2):51-65. https://doi.org/10.1002/bdd.1952

Watanabe T, Katsumata N, Sasaki Y, et al. A multicentre phase II trial of Xeloda (capecitabine) in patients with docetaxel-refractory advanced/metastatic breast cancer. Proceedings of the American Society of Clinical Oncology. 2001. 20:61b. Abstract 1991

Weigelt B, Peterse JL, van't Veer L. Breast cancer metastasis: markers and models. Nat Rev Cancer. 2005;5(8):591-602. https://doi.org/10.1038/nrc1670

Wolf SL, Qin R, Menon SP, et al. Placebo-controlled trial to determine the effectiveness of a urea/lactic acid-based topical keratolytic agent for prevention of capecitabine-induced hand-foot syndrome: North Central Cancer Treatment Group Study N05C5. J Clin Oncol. 2010;28(35):51825187. https://doi.org/10.1200/JCO.2010.31.1431 